\title{
TORCICOLO ESPASMÓDICO E MENINGEOMA FRONTAL
}

\author{
EDWARD R. TONHOLO-SILVA * - ELIZABETH M. A. BARASNEVÍCIUS QUAGLIATO **
}

RESUMO - Foi estudada o caso de um paciente de 57 anos com meningeoma frontal esquerdo, cuja única manifestação clínica foi torcicolo espasmódico contralateral. Há poucos casos relatados na literatura de lesões frontais levando a movimentos involuntários, sendo analisada a possibilidade de uma desconexão funcional entrei o córtex frontal e o complexo estriato-lentículo-talâmico.

Spasmodic torticollis and frontal meningioma.

SUMMARY - A 57 year-old man developed a spasmodic torticollis with involuntary deviation of the head to the right-side. He had a left paramedian frontal meningioma. The association of spasmodic torticollis and other movement disorders has been reported with contralateral lesions in the basal ganglia. Its occurrence in association to a frontal lesion appears to be much less frequent suggesting a possible disorder of frontostriatal connections.

0 torcicolo espasmódico (TE) é conceitudo clinicamente como uma contração involuntária dos músculos do pescoço, levando a movimentos mantidos e/ou posição anormal da cabeça acometendo com maior frequência os músculos osternocleidomastóideo e trapézio. Relaciona-se a uma variedade de fatores etiopatogênicos, alguns ainda não conhecidos 23, postulando-se até mesmo origem psicogênica 3. Atualmente, na maioria dos casos, já existem evidências farmacológicas e experimentais implicando numa disfunção orgânica do sistema extrapiramidal $*^{*}$, sendo considerado uma distonia focal ${ }^{12}$. Geralmente tem início na idade adulta, principalmente na quarta e quinta décadas ${ }^{\wedge} M$, Alguns pacientes têm, associadas, outras desordens do movimento tais como tremor, cãibra do escrivão ou outros quatros distônicos. Foram descritos casos com lesões localizadas no lobo frontal, apesar de haver outros locais de acometimento.

Descrevemos um caso cuja lesão acometia o lobo frontal esquerdo e analisamos as implicações etiopatogênicas de lesão frontal originando TE.

\section{OBSERVAÇÃO}

MP, registro HC 224570-7, paciente do sexo masculino, com 57 anos de idade, não judeu, com queixa de cervicalgia há dois anos, à movimentação da cabeça para esquerda (E), mantendo postura sustentada do pescoço para a direita (D). Esporadicamente apresentava dificuldade de movimentar o membro superior $D$, por ocasião da realização de movimentos mais finos, sem alterações da escrita. Negava outras queixas. Tabagista desde a adolescência. Exame físico geral $-\mathbf{P A}=\mathbf{1 7 0 / 1 1 0} \mathrm{mmHg}$, detectada na internação; sem outras alterações. Exame neurológico - consciente, orientado no tempo e espaço, contactuando adequadamente, psiquismo íntegro, movimentos musculares ativos preservados, exceto diminuição da movimentação da musculatura cervical para a $\mathbf{E}$, hipertonia do músculo esternocleidomastóideo $\mathrm{D}$

Departamento de Neurologia da Faculdade de Ciências Médicas/UNICAMP: * Médico Residente em Neurologia Clínica; ** Professor Assistente Doutor.

Dr. Edward R. Tonholo-Silva - Rua Julio Mesquita 295 - 17500 Marília SP - Brasil. 
e postura de rotação do pescoço para a D; restante do exame normal. Exames complementares - Hemograma, eoagulograma, VHS, urina I, provas de função hepática, glicemia, $\mathrm{Na}+, \mathrm{K}+»$ uréia, creatinina, ECG e EEG, dentro da normalidade. RX simples de crânio normal e, no de coluna cervical osteófitos em C5 e C6. TCC: lesão hiperdensa, de cerca de 4 cm de diámetro, paramediana, captadora de contraste, na região frontal $\mathrm{E}^{\wedge}$ sugestiva de meningeoma (Fig. IA). Estudo angiográfico cerebral: lesão que retinha contraste, compatível a meningeoma frontal E. Medicado com clortalidona, $50 \mathrm{mg} / \mathrm{d}$, com controle adequado da hipertensão. O paciente recusou ser submetido a cirurgia para exérese do tumor.

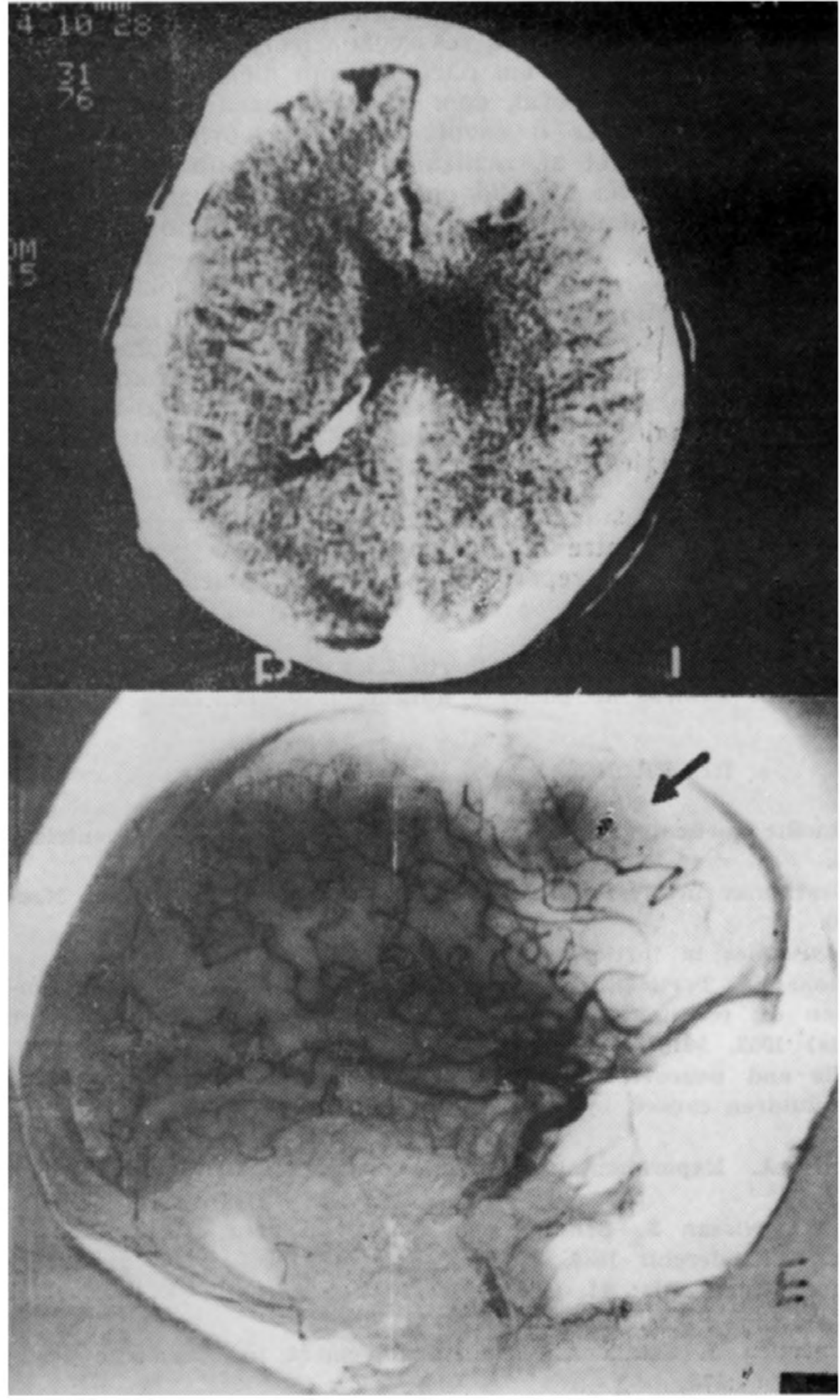

Fig. 1 - Gaso MP. Em A (no alto), TCC cum contraste mostrando hiperdensidade parassagital frontal esquerda.

Em $B$ (em baixo), exame angiografico carotideo mostrando lesâo bem definida. captadora de contraste, vas cularizada, na regiäo frontal esquerda.

\section{COMENTÁRIOS}

Há muitas discussões quanto à origem do TE, postulando-se etiologia psíquica mas, atualmente, tenta-se explicar por disfunção do sistema extrapiramidal a gênese do TE. Várias autópsias de pacientes com TE mostraram lesões nos núcleos da base, mas o TE fazia parte de distonia generalizada, não sendo estabelecida a presença de lesão específica responsável pelo TE 21. Em animais, o TE pode ser produ- 
zido por lesões em tronco cerebral 17. Também foi encontrado em pacientes com siringomielia ou tumor medular n e, também, associado a cisto colóide do terceiro ventrículo. Há possibilidade de algum envolvimento do sistema vestibular na gênese do TE:Bronstein e Rudge encontraram anormalidade no sistema vestibular em $70 \%$ dos casos com TE, na ausência de outros sintomas otológicos e neurológicos². Outros casos de TE estão asspciados a problemas musculares congênitos 5,19,20 hérnia de hiato 8 e, em crianças, pode ser causado por hemi-aplasia ou hipoplasia do atlas 6 .

No presente caso, o paciente apresentava meningeoma frontal esquerdo, cuja única manifestação clínica foi o TE contralateral. Na literatura, há poucos casos relatados de $\mathrm{TE}$ com lesão frontal. David et al relataram caso de $\mathrm{TE}$ associado a cicatriz frontal pós-traumática, que desapareceu após ressecção cirúrgica ${ }^{4}$ Meyrignac et al., mais recentemente, descreveram o caso de um paciente com meningeoma frontal esquerdo causando cãibra do escrivão contralateral, com evolução para TE e distonia axial !6. Há estudos na tentativa de mostrar o envolvimento do córtex frontal na gênese de movimentos involuntários. Kaste et al. realizaram estudo com pneumoencefalografia, mostrando a ocorrência de atrofia cortical em áreas fronto-parietais corticais em $77 \%$ dos pacientes com TE estudados, sugerindo que a lesão no TE esteja localizada no córtex motor e nas áreas associativas com os núcleos da base ${ }^{10}$. Marsden et al. suspeitaram de disfunção frontal para as hemidistonias, ou distonias focais, devido à perda do controle inibitório normalmente exercido pelo estriato e núcleo lenticular, com um relê talâmico sobre a área motora frontal e área motora suplementar 13. No estudo de pacientes com hemidistonias, Pettigrew e Jankovic conclúram que o movimento involuntário seja devido à desconexão estriato-talâmica, com preservação do trato cortico-espinhal 17. Já no estudo de van Hoof et al., não foram encontrados dados consistentes de lesão cerebral orgânica 22.

A análise do presente caso permite supor que a lesão frontal pode, de algum modo, perturbar a interligação funcional entre os núcleos da base e áreas frontais pré-motora e motora suplementar, levando a repercussão sobre as conexões estriato-lentículo-talâmicas.

Agradecimentos - Agradecemos aos colegas Drs. Alberto Luiz Cunha da Costa e Marcelo Annes, pela ajuda nas avaliações clínicas do paciente durante a internação.

\section{REFERÊNCIAS}

1. Avman N, Arasil E. Spasmodic torticollis due to colloid cyst of the third ventricle. Acta Neurochir 1969, 21:266.

2. Bronstein AM, Rudge P. Vestibular involvement in spasmodic torticollis. J Neurol Neurosurg Psychiat 1986, 49:290.

3. Cleveland SE, Personality dynamics in torticollis. J Nerv Ment Dis 1961, 129:150.

4. Pavid $\mathrm{M}$, Hécaen $\mathrm{H}$, Constans J. Torticollis spasmodique consécutif à une lesion corticate traumatique: discussion du resultat favorable obtenu après excision de la lésion corticale. Rev Neurol (Paris) 1952, 86:57.

5. Dick P. Spasmodic torticollis and neurovascular compression. J Neurosurg 1986, 64 : 686.

6. Dubousset J. Torticollis in children caused by congenital anomalies of the atlas. J Bone Joint Surg 1986, 68A : 178.

7. Foltz EL, Knopp LM, Ward AA. Experimental spasmodic torticollis. J Neurosurg 1959, 16:55.

8. Hadari A, Azizi E, Lernau O, Nissan S. Sandifer's syndrome: a rare complication of hiatal hernia. A case report. Z Kinderchir 1984, 39:202.

9. Herz E, Glaser GH. Spasmodic torticollis: II. Clinical evaluation. Arch Neurol Psychiat 1949, 61:227.

10. Kaste M, Ilvanainen M, Juntunen J, Setala A. Brain involvement in spasmodic torticollis. Acta Neurol Scand 1981, 63:373.

11. Kiwak KJ, Deray MJ, Shields WD. Torticollis in three children with syringomielia and spinal cord tumor. Neurology 1983, 33:946.

12. Marsden CD. Dystonia: the spectrum of the disease. Res Publ Ass Res Nerv Ment Dis $1976,55: 351$.

13. Marsden CD, Obeso JA, Zarranz JJ, Lang AE. The anatomical basis of symptomatic hemidystonia. Brain 1985, 108:463.

14. Matthews WB, Beasley P, Parry-Jones E, Garland G. Spasmodic torticollis: a combined clinical study. J Neurol Neurosurg Psychiat 1978, 41 : 485.

15. McGeer EG, McGeer PL. The dystonias. Can J Neurol Sci 1988, 15:447. 
16. Meyrignac C, Kéravel Y, Boulu F, Nguyen JP, Degos JD. Crampe des écrivains et méningeoma frontal gauche. Rev Neurol (Paris) 1988, 144:378.

17. Pettigrew LC, Jankovic J. Hemidystonia: a report of 22 patients and a review of the literature. J Neurol Neurosurg Psychiat 1985, 48:650.

18. Podivinsky F. Torticollis. In Vinken PJ, Bruyn GW (eds): Handbook of Clinical Neurology. Amsterdam: North-Holland, 1968, Vol 6, p 567.

19. Sarnat HB, Morrissy RT. Idiopatic torticollis: sternocleidomastoid myopathy and accessory neuropathy. Muscle \& Nerve 1981, $4: 374$.

20. Shima F, Fukui M, Matsubara T, Kitamura K. Spasmodic torticollis caused by vascular compression of the spinal accessory root. Surg Neurol 1986, 26:431.

21. Tarlov E. On the problem of the pathology of spasmodic torticollis in man. $J$ Neurol Neurosurg Psychiat 1970, $33: 457$.

22. van Hoof JJM, Horstink MWI, Berger HJC, van Spaendonck KPM, Cools AR. Spasmodic torticollis: the problem of pathophysiology and assessment. J Neurol 1987, 234:322.

23. Zeman W, Dyken P. Dystonia musculorum deformans. In Vinken PJ, Bruyn GW (eds) : Handbook of Clinical Neurology. Amsterdam: North-Holland, 1968, Vol 6, p 529. 